\title{
Actualización de la distribución geográfica de la serpiente índigo Drymarchon corais (Boie, 1827) en la Amazonía ecuatoriana
}

\author{
Manuel R. Dueñas ${ }^{*}$, Jorge H. Valencia ${ }^{2,3}$, Daniela Franco-Mena ${ }^{4}$ \\ IInstituto Nacional de Biodiversidad, Museo Ecuatoriano de Ciencias Naturales, División de \\ Herpetología, Rumipamba 341 y Av. de los Shyris. Casilla 17-07-8976, Quito, Ecuador. \\ 2Laboratorio de Anfibios y Reptiles, Fundación Herpetológica Gustavo Orcés, Quito, Ecuador. \\ ${ }^{3}$ Red de Biología y Conservación de Vertebrados, Instituto de Ecología, carretera antigua a \\ Coatepec, 91070, Xalapa, Veracruz, México. \\ ${ }^{4}$ Laboratorio de Biología Evolutiva, Instituto Biósfera, Colegio de Ciencias Biológicas y Ambientales, \\ Universidad San Francisco de Quito USFQ, Quito, Ecuador.. \\ *Autor para correspondencia/Corresponding author: duenastmanuel@hotmail.com.
}

\section{Update of the geographical distribution of the indigo snake Drymarchon corais (Boie, 1827) in the Ecuadorian Amazon}

\begin{abstract}
Resumen
Se presenta la actualización de la distribución geográfica de la serpiente índigo Drymarchon corais en la Amazonía ecuatoriana. Los datos presentados fueron obtenidos de la revisión de registros históricos, colección de especímenes en campo, revisión de especímenes en colecciones zoológicas, información de investigadores de campo y revisión de bases de datos de libre acceso. El estudio evidencia la presencia de la especie en localidades y rangos altitudinales que anteriormente eran desconocidos. Además, se presentan datos de historia natural que permiten ampliar el conocimiento sobre la ecología de especie.
\end{abstract}

Palabras clave: Distribución geográfica, serpiente índigo, Drymarchon, Amazonía, Ecuador.

\section{Abstract}

Editado por /

Edited by:

Elisa Bonaccorso

Recibido /

Received:

01/17/2021

Aceptado /

Accepted:

07/07/2021

Publicado en línea / Published online: $15 / 12 / 2021$

This article presents an update of the geographic distribution of the indigo snake Drymarchon corais in the Ecuadorian Amazon. The data presented was obtained from the review of historical records, collection of specimens in the field, review of specimens in zoological collections, information from field researchers, and review of open access databases. The study shows the presence of the species in localities and in altitudinal ranges that were previously unknown. In addition, the article presents natural history data that expands the knowledge about the species' ecology.

Keywords: Geographical distribution, indigo snake, Drymarchon, Amazon, Ecuador. 


\section{INTRODUCCIÓN}

En el mundo, los bosques neotropicales albergan la mayor cantidad de especies [1,2]. Dentro de estos, en Sudamérica, la Amazonía constituye una de las regiones biogeográficas más grandes del planeta y en Ecuador representa la región de mayor extensión con aproximadamente $120.000 \mathrm{Km}^{2}$ [3]. En este país, tan solo el Bosque Húmedo Tropical Amazónico agrupa el 31,57\% de la riqueza de especies de reptiles (156 especies) [4]. Las investigaciones herpetológicas desarrolladas en la Amazonía ecuatoriana en los últimos años han generado la descripción de nuevas especies (e.g., $[5,6,7])$, extensiones de rango de distribución [8] y nuevos registros para el país [9,10,11]. Lo expuesto señala la importancia de investigar y presentar la actualización de los patrones de distribución de especies que, posteriormente, permitirán plantear estrategias de conservación.

Las serpientes neotropicales del género Drymarchon (Fitzinger, 1843) comprenden serpientes medianas a grandes que alcanzan una longitud total de $327 \mathrm{~cm}$ [12] y presentan un cuerpo predominantemente robusto [13]. Las especies de este género tienen hábitos terrestres y una dieta generalista que incluye anfibios, saurios, serpientes, aves, huevos y pequeños mamíferos como roedores y marsupiales [14-17]. Se ha documentado que los procesos de alimentación de estas especies se desarrollan de manera activa durante el día en ambientes abiertos y boscosos $[16,18]$. El rango de distribución del género Drymarchon se extiende desde el Sureste de los Estados Unidos hasta el Norte de Argentina, incluida gran parte de México y América Central [13-20].

Actualmente, las cinco especies descritas en el género son: Drymarchon caudomaculatus, D. corais, D. couperi, D. margaritae y D. melanurus [21]. D. corais se distribuye desde México hasta Paraguay; incluyendo Guyana, Venezuela, Colombia, Ecuador, Perú, Bolivia y Brasil $[13,21]$. En Ecuador, los escasos registros de D. Corais señalan que está presente al Este de los Andes en los ecosistemas de tierras bajas amazónicas [4]. Previamente, esta especie se ha reportado en las provincias de Orellana, Napo y Morona Santiago $[4,22,23,24]$, a pesar de que varios autores la reportan de manera general en la región Amazónica [25-32], y que ha sido introducida accidentalmente en la provincia de Pichincha [33]. En este contexto, el objetivo de este trabajo es presentar una actualización de la distribución geográfica y elevacional de Drymarchon corais en la Amazonía ecuatoriana.

\section{MATERIALES Y MÉTODOS}

Los registros de localidades de presencia de Drymarchon corais se obtuvieron de varias fuentes: 1) especímenes depositados en los Museos de la Escuela Politécnica Nacional del Ecuador (MEPN), Quito, Ecuador; Laboratorio de Anfibios y Reptiles, Fundación Herpetológica Gustavo Orcés (FHGO), Quito, Ecuador; 2) especímenes del American Museum of Natural History (AMNH), Los Ángeles County Museum (LACM), Museum Comparative of Zoology (MCZ), University of Kansas (KU), a través de las plataformas digitales GBIF, Vernet and iNaturalist; 3) observaciones de campo y registros fotográficos de varios investigadores. Todos los registros fueron georreferenciados, las coordenadas fueron transformadas a grados, minutos y segundos en la plataforma virtual Google Earth (versión 7.3.2). El mapa de localidades fue elaborado en el software ArcMap (versión 10.4) en una escala de 1: 1.000.000. 


\section{RESULTADOS}

Se compiló un total de 33 registros de individuos de Drymarchon corais (Tabla 1), los cuales corresponden a 22 diferentes localidades, 18 de los cuales son nuevas para la Amazonía ecuatoriana (Fig. 1). Diez de estos registros fueron obtenidos de la literatura y bases de datos digitales (i.e., $[4,18,21,22,23,24,27,28,29,30,34]$ ), nueve de información de especímenes depositados en museos de historia natural y los restantes de información proporcionada por diferentes investigadores.

Tabla 1. Registros históricos y nuevos de la serpiente índigo Drymarchon corais (Boie, 1827) en la Amazonía ecuatoriana (*Registros históricos; †Registro fotográfico de la especie; NA, no aplica porque no tiene un espécimen asociado depositado en museos).

\begin{tabular}{|c|c|c|c|c|}
\hline $\mathbf{N}^{0}$ & $\begin{array}{l}\text { Código } \\
\text { Museo }\end{array}$ & $\begin{array}{l}\text { Provincia, localidad, } \\
\text { coordenadas, altitud }\end{array}$ & Colector, observador & $\begin{array}{l}\text { Fecha de } \\
\text { registro }\end{array}$ \\
\hline 1 & MCZ 154059 & $\begin{array}{c}\text { Sucumbíos, Limoncocha } \\
\left(0^{\circ} 23^{\prime} 49,15^{\prime \prime} \text {; } 76^{\circ} 36^{\prime} 37,51^{\prime \prime} \mathrm{O} ; 252\right. \\
\text { m s.n.m. })\end{array}$ & $\begin{array}{c}\text { Charles M. Fugler } \\
\text { (Museo Comparativo de } \\
\text { Zoología) }\end{array}$ & $21 / 01 / 1929 *$ \\
\hline 2 & LACM 73364 & $\begin{array}{c}\text { Sucumbíos, Limoncocha } \\
\left(0^{\circ} 23^{\prime} 49,15^{\prime \prime} \text {; } 76^{\circ} 36^{\prime} 37,51^{\prime \prime} \mathrm{O} ; 252\right. \\
\text { m s.n.m. })\end{array}$ & $\begin{array}{l}\text { W.R. Heyer (Museo del } \\
\text { Condado de Los Ángeles) }\end{array}$ & 03/08/1971 * \\
\hline 3 & KU 158781 & $\begin{array}{l}\text { Orellana, El Coca }\left(0^{\circ} 24^{\prime} 00^{\prime \prime} \text { S; }\right. \\
77^{\circ} 0^{\prime} 00^{\prime \prime} \text { O; } 267 \text { m s.n.m.) }\end{array}$ & Universidad de Kansas & $23 / 04 / 1975 *$ \\
\hline 4 & KU 183514 & $\begin{array}{c}\text { Sucumbíos, Limoncocha } \\
\left(0^{\circ} 23^{\prime} 49,15^{\prime \prime} \text { S; } 76^{\circ} 36^{\prime} 37,51^{\prime \prime} \mathrm{O} ; 252\right. \\
\text { m s.n.m.) }\end{array}$ & Universidad de Kansas & 21/03/1979* \\
\hline 5 & MCZ 107658 & $\begin{array}{l}\text { Morona Santiago (sin datos de } \\
\text { localidades ni coordenadas) }\end{array}$ & $\begin{array}{l}\text { Museo Americano de } \\
\text { Historia Natural }\end{array}$ & Desconocido* \\
\hline 6 & MCZ 107659 & $\begin{array}{l}\text { Morona Santiago (sin datos de } \\
\text { localidades ni coordenadas) }\end{array}$ & $\begin{array}{l}\text { Museo Americano de } \\
\text { Historia Natural }\end{array}$ & Desconocido* \\
\hline 7 & MEPN 8830 & Sin datos de localidad ni colección & Ana Almendáriz & $1991 *$ \\
\hline 8 & NA & $\begin{array}{l}\text { Napo (sin datos de localidades ni } \\
\text { coordenadas) }\end{array}$ & Pérez-Santos y Moreno & $1991 *$ \\
\hline 9 & NA & $\begin{array}{l}\text { Napo, Tena, Ahuano ( } 1^{\circ} 4^{\prime} 0,84^{\prime \prime} \text {; } \\
77^{\circ} 37^{\prime} 12,72^{\prime \prime} \mathrm{O} ; 427 \text { m s.n.m.) }\end{array}$ & Diego Cisneros-Heredia & 07/11/1999 \\
\hline 10 & NA & $\begin{array}{l}\text { Orellana, Estación de Biodiversidad } \\
\text { Tiputini }\left(0^{\circ} 38^{\prime} 15,72^{\prime \prime} \text { S; } 76^{\circ} 8^{\prime} 59,64^{\prime \prime}\right. \\
\text { O; } 216 \text { m s.n.m.) }\end{array}$ & Diego Cisneros-Heredia & 29/12/1999 \\
\hline 11 & NA & $\begin{array}{c}\text { Orellana, Estación de Biodiversidad } \\
\text { Tiputini }\left(0^{\circ} 38^{\prime} 22,20^{\prime \prime} \text { S; } 76^{\circ} 9^{\prime} 38,16^{\prime \prime}\right. \\
\text { O; } 223 \text { m s.n.m. })\end{array}$ & Diego Cisneros-Heredia & $12 / 06 / 2000$ \\
\hline 12 & NA & $\begin{array}{l}\text { Sucumbíos, Nueva Juventud } \\
\left(0^{\circ} 4^{\prime} 43,00^{\prime \prime} \text { S; } 76^{\circ} 24^{\prime} 9,00^{\prime \prime} 0 ; 249 \mathrm{~m}\right. \\
\text { s.n.m.) }\end{array}$ & Diego Cisneros-Heredia & $15 / 07 / 2000$ \\
\hline
\end{tabular}




\begin{tabular}{|c|c|c|c|c|c|}
\hline 17 & $\mathrm{~N}^{\circ}$ & $\begin{array}{l}\text { Código } \\
\text { Museo }\end{array}$ & $\begin{array}{l}\text { Provincia, localidad, } \\
\text { coordenadas, altitud }\end{array}$ & Colector, observador & $\begin{array}{l}\text { Fecha de } \\
\text { registro }\end{array}$ \\
\hline \multirow[t]{17}{*}{$\begin{array}{l}\text { avances } \\
\text { encienciase } \\
\text { ingenierías }\end{array}$} & 13 & FHGO 5742 & $\begin{array}{c}\text { Orellana, Parque Nacional Yasuní } \\
\left(0^{\circ} 40^{\prime} 13,68^{\prime \prime} \text { S; } 76^{\circ} 23^{\prime} 58,20^{\prime \prime} \mathrm{O} ; 245\right. \\
\text { m s.n.m.) }\end{array}$ & $\begin{array}{l}\text { Morley Read, Edwin } \\
\text { Carillo, Silvia Aldás }\end{array}$ & $15 / 11 / 2005+$ \\
\hline & 14 & NA & $\begin{array}{c}\text { Orellana, Estación de Biodiversidad } \\
\text { Tiputini }\left(0^{\circ} 37^{\prime} 5^{\prime \prime} \text { S; } 76^{\circ} 10^{\prime} 19^{\prime \prime} \mathrm{O} ; 234\right. \\
\text { m s.n.m.) }\end{array}$ & Diego Cisneros-Heredia & $2006 *$ \\
\hline & 15 & NA & $\begin{array}{c}\text { Morona Santiago, Cusuime } \\
\left(2^{\circ} 48^{\prime} 7,99^{\prime \prime} \text { S } 77^{\circ} 38^{\prime} 30,84^{\prime \prime} \mathrm{O} ; 215\right. \\
\text { m s.n.m. })\end{array}$ & Mauricio Ortega-Andrade & $2010^{*}$ \\
\hline & 16 & FHGO 9091 & $\begin{array}{c}\text { Sucumbíos, Comunidad } 13 \text { de } \\
\text { Marzo }\left(0^{\circ} 12^{\prime} 2^{\prime \prime} \mathrm{N} ; 76^{\circ} 33^{\prime} 11^{\prime \prime} \mathrm{E} ; 250\right. \\
\text { m s.n.m. })\end{array}$ & Manuel R. Dueñas & $15 / 01 / 2012+$ \\
\hline & 17 & NA & $\begin{array}{c}\text { Sucumbíos, Limoncocha } \\
\left(0^{\circ} 22^{\prime} 48,30^{\prime \prime} \text { S; } 76^{\circ} 35^{\prime} 36,73^{\prime \prime} \mathrm{O} ; 251\right. \\
\text { m s.n.m. })\end{array}$ & Jaime Culebras & 04/02/2012 \\
\hline & 18 & NA & $\begin{array}{c}\text { Zamora Chinchipe, río } \\
\text { Bombuscara ( } 4^{\circ} 04^{\prime} 04,61^{\prime \prime} \text { S; } \\
78^{\circ} 56^{\prime} 23,27^{\prime \prime} \text { O; } 937 \text { m s.n.m.) }\end{array}$ & Darwin Núñez & $25 / 04 / 2012$ \\
\hline & 19 & NA & $\begin{array}{c}\text { Zamora Chinchipe, vía al Jenairo } \\
\left(4^{\circ} 2^{\prime} 57,29^{\prime \prime} \text { S; } 78^{\circ} 58^{\prime} 0,20^{\prime \prime} 0 ; 1048\right. \\
\text { m s.n.m. })\end{array}$ & Darwin Núñez & 07/08/2014 \\
\hline & 20 & NA & $\begin{array}{c}\text { Zamora Chinchipe, Zamora } \\
\left(4^{\circ} 3^{\prime} 13,01^{\prime \prime} \text { S; } 78^{\circ} 56^{\prime} 27,21^{\prime \prime} \mathrm{O} ; 960\right. \\
\text { m s.n.m. })\end{array}$ & Darwin Núñez & 15/01/2015 \\
\hline & 21 & NA & $\begin{array}{c}\text { Zamora Chinchipe, vía a Jamboe } \\
\left(4^{\circ} 3^{\prime} 26,11^{\prime \prime} \text { S } 78^{\circ} 55^{\prime} 44,23^{\prime \prime} \mathrm{O} ; 903\right. \\
\text { m s.n.m.) }\end{array}$ & Darwin Núñez & 22/03/2015 \\
\hline & 22 & NA & $\begin{array}{l}\text { Orellana, Tiputini ( } 0^{\circ} 38^{\prime} 10,32^{\prime \prime} \mathrm{S} \text {; } \\
\quad 76^{\circ} 9^{\prime} 51,99^{\prime \prime} \mathrm{O} ; 218 \mathrm{~m} \text { s.n.m.) }\end{array}$ & Jaime Culebras & $10 / 11 / 2016$ \\
\hline & 23 & NA & $\begin{array}{l}\text { Napo, Ahuano (1 } 1^{\circ} 3^{\prime} 13,46^{\prime \prime} S_{;} \\
77^{\circ} 31^{\prime} 51,34^{\prime \prime} \text { O; } 356 \text { m s.n.m.) }\end{array}$ & Jaime Culebras & $14 / 06 / 2017 \dagger$ \\
\hline & 24 & NA & $\begin{array}{c}\text { Orellana, Nueva Esperanza } \\
\left(0^{\circ} 29^{\prime} 57,48^{\prime \prime} \text { S } 76^{\circ} 45^{\prime} 53,80^{\prime \prime} 0 ; 261\right. \\
\text { m s.n.m. })\end{array}$ & Diego Naranjo, iNaturalist & 19/06/2017† \\
\hline & 25 & NA & $\begin{array}{l}\text { Pastaza, Kapawi (2³2'28,24"S; } \\
76^{\circ} 51^{\prime} 30,96^{\prime \prime} \text { O; } 249 \text { m s.n.m.) }\end{array}$ & Jaime Culebras & 07/09/2017 \\
\hline & 26 & NA & $\begin{array}{l}\text { Orellana, Chiru Isla }\left(0^{\circ} 35^{\prime} 38^{\prime \prime} \text {; ; } 76^{\circ}\right. \\
\text { 4'30"O; } 232 \text { m s.n.m.) }\end{array}$ & Luis Oyagata & 06/05/2018 t \\
\hline & 27 & NA & $\begin{array}{c}\text { Zamora Chinchipe, Congüime } \\
\left(4^{\circ} 2^{\prime} 53,34^{\prime \prime} \text { S; } 78^{\circ} 38^{\prime} 9,12^{\prime \prime} \mathrm{O} ; 896 \mathrm{~m}\right. \\
\text { s.n.m.) }\end{array}$ & Darwin Núñez & 13/07/2018 \\
\hline & 28 & NA & 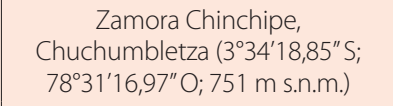 & Darwin Núñez & $17 / 09 / 2018$ \\
\hline & 29 & NA & $\begin{array}{c}\text { Morona Santiago, Cangaime } \\
\left(2^{\circ} 47^{\prime} 15^{\prime \prime} \text { S; } 77^{\circ} 31^{\prime} 33^{\prime \prime} \mathrm{O} ; 224 \mathrm{~m}\right. \\
\text { s.n.m. })\end{array}$ & Axel Marchelie & $27 / 05 / 2019+$ \\
\hline
\end{tabular}




\begin{tabular}{|c|c|c|c|c|c|}
\hline 17 & $\mathrm{~N}^{\circ}$ & $\begin{array}{l}\text { Código } \\
\text { Museo }\end{array}$ & $\begin{array}{l}\text { Provincia, localidad, } \\
\text { coordenadas, altitud }\end{array}$ & Colector, observador & $\begin{array}{l}\text { Fecha de } \\
\text { registro }\end{array}$ \\
\hline \multirow[t]{4}{*}{$\begin{array}{l}\text { avances } \\
\text { encieciase } \\
\text { ingenierias }\end{array}$} & 30 & NA & $\begin{array}{c}\text { Orellana, Nuevo Rocafuerte } \\
\left(0^{\circ} 55^{\prime} 22,36^{\prime \prime} \text { S; } 75^{\circ} 23^{\prime} 57,64^{\prime \prime} \mathrm{O} ; 183\right. \\
\text { m s.n.m.) }\end{array}$ & Mike Tidwell, iNaturalist & 07/08/2019† \\
\hline & 31 & NA & $\begin{array}{l}\text { Napo, Ahuano ( } 1^{\circ} 04^{\prime} 36,50^{\prime \prime} S_{;} \\
77^{\circ} 31^{\prime} 40,53^{\prime \prime} \mathrm{O} ; 433 \text { m s.n.m.) }\end{array}$ & Amazoonico, iNaturalist & $16 / 02 / 2020+$ \\
\hline & 32 & NA & $\begin{array}{c}\text { Morona Santiago, Guayabal } \\
\left(3^{\circ} 25^{\prime} 2^{\prime \prime} \text { S; } 78^{\circ} 33^{\prime} 38^{\prime \prime} \mathrm{O} ; 810 \mathrm{~m}\right. \\
\text { s.n.m. })\end{array}$ & Ángel Vásquez & 09/04/2020† \\
\hline & 33 & NA & $\begin{array}{c}\text { Zamora Chinchipe, Nuevo Paraíso } \\
\left(4^{\circ} 28^{\prime} 03,93^{\prime \prime} \text { S; } 78^{\circ} 41^{\prime} 26,95^{\prime \prime} \mathrm{O} ; 1110\right. \\
\text { m s.n.m.) }\end{array}$ & Twstiles, iNaturalist & $20 / 04 / 2020 \dagger$ \\
\hline
\end{tabular}

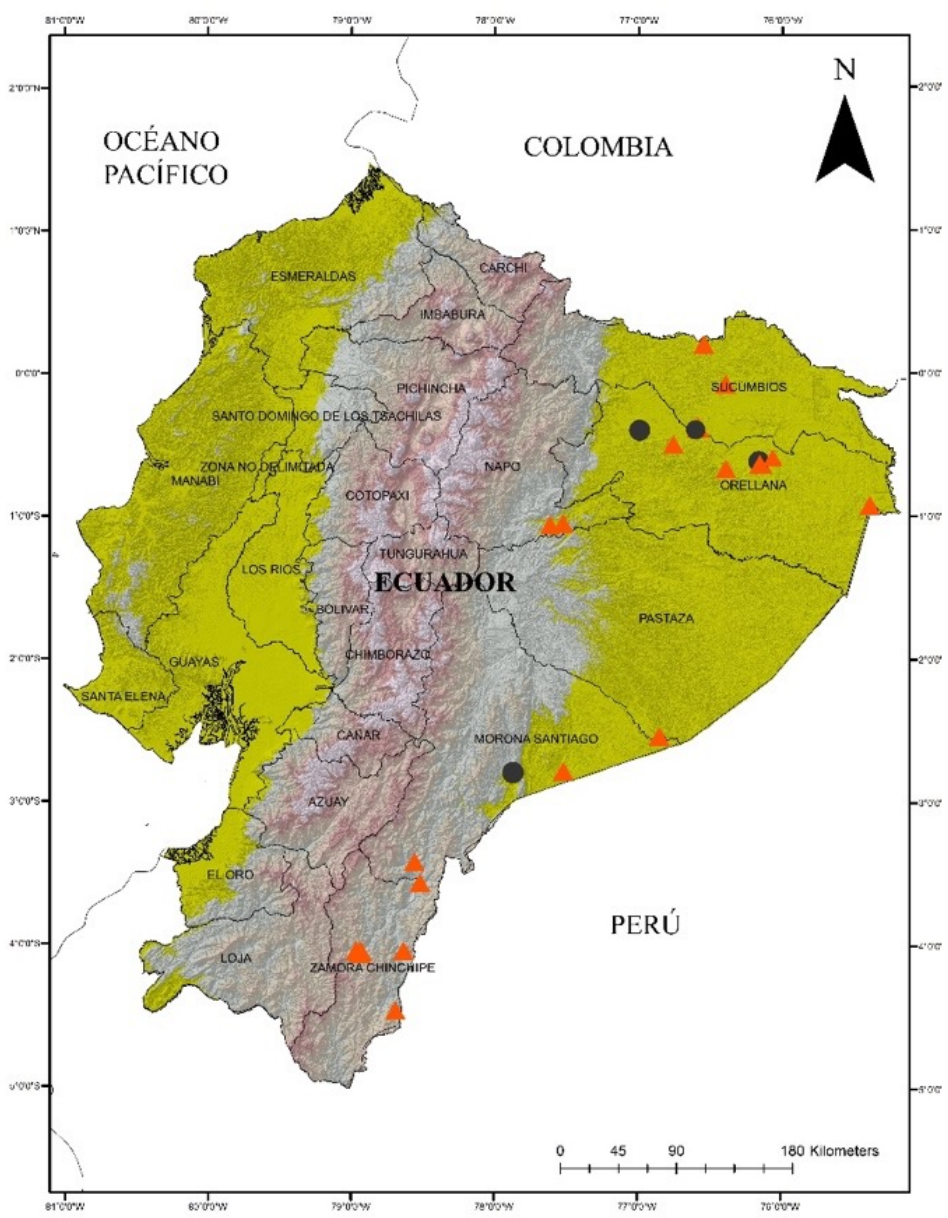

Figura 1. Registros de la serpiente índigo Drymarchon corais en la Amazonía ecuatoriana. Círculos negros: registros históricos, Triángulos anaranjados: nuevos registros. 


\section{REGISTROS HISTÓRICOS}

Tres especímenes (MCZ 154059, LACM 73364, KU 183514) fueron encontrados en los alrededores de laguna de Limoncocha ( $0^{\circ} 23^{\prime} 49,15^{\prime \prime}$ S; $76^{\circ} 36^{\prime} 37,51^{\prime \prime}$ W; 229 m s.n.m.), cantón Shushufindi, provincia de Sucumbíos. Estos fueron recolectados el 21 de enero de 1929 por Charles M. Fugler, el 21 de marzo de 1979 por Ronald Heyer y 23 de abril de 1975 por William Duellman, respectivamente. Un espécimen (KU 158781) fue identificado en la ciudad de El Coca (i.e., Puerto Francisco de Orellana, provincia de Orellana) $\left(0^{\circ} 24^{\prime} 00^{\prime \prime}\right.$ S; $77^{\circ} 0^{\prime} 00^{\prime \prime}$ W; 267 m s.n.m.) el 23 de abril de 1975. Dos especímenes adicionales (AMNH $107658,107659)$ fueron colectados en la provincia de Morona Santiago sin localidad específica. Finalmente, un espécimen (MEPN 8830), sin localidad fue colectado en la Amazonía de Ecuador.

Algunos autores reportan a Drymarchon corais en la Amazonía de Ecuador [4,22,23,24,26], aunque sus registros no están respaldados por material voucher (especímenes o fotografías). Por ejemplo, Cisneros-Heredia [22] reportó un individuo en la Estación de Biodiversidad Tiputini (EBT), provincia de Orellana, pero no reporta evidencia fotográfica o información de especímenes. M. Ortega [24] reportó un individuo en el sector de Cusuime, provincia de Morona Santiago sin evidencia fotográfica ni colección de un espécimen voucher. La información acerca de la distribución de Drymarchon corais señala que es una especie que se distribuye en la Amazonía ecuatoriana $[4,18,22,23,26,32]$ y que accidentalmente ha sido introducida en la provincia de Pichincha [33]. En la lista reptiles de Ecuador, Almendáriz [30] reporta a Drymarchon corais en la Amazonía de Ecuador (sin detalle de localidad). Del mismo modo, Pérez-Santos y Moreno [23] en su monografía de Serpientes de Ecuador mencionan que la especie está presente en la provincia de Napo, sin mayor detalle de localidades.

\section{NUEVOS REGISTROS}

Un espécimen joven de Drymarchon corais (FHGO 5742) fue colectado por Morley Read, Edwin Carrillo y Silvia Aldás (Fig. 2A, 2B) el 15 de noviembre de 2005 en el Parque Nacional Yasuní, vía a CPF (040'13,68"S; 76²3'58,20" O; 245 m s.n.m.), cantón Aguarico, provincia de Orellana. Otro espécimen (FHGO 9091) hembra adulta (Fig. 2C, 2D), fue colectado por Manuel R. Dueñas el 15 de enero de 2012, a las 09:32 h en la localidad 13 de Marzo (0¹2'2" N; 76³3'11" W; 250 m s.n.m.), cantón Pacayacu, provincia de Sucumbíos (Fig. 1). Ambos especímenes (FHGO 5742, 9091) fueron encontrados muertos. El primero atropellado en la carretera, y el segundo después del ataque de pobladores locales. Adicionalmente, se encontró que el espécimen FHGO 9091, contenía en su estómago restos de un sapo de la caña Rhinella marina (Fig. 3A). 

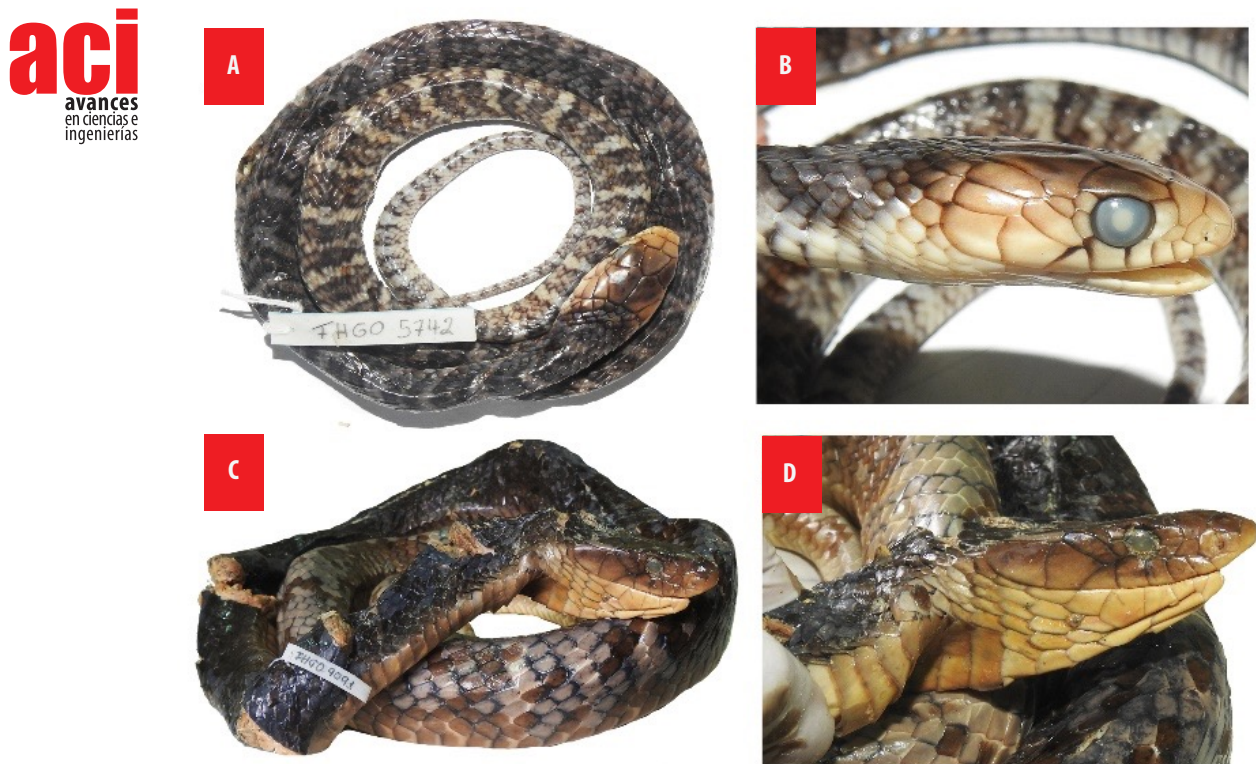

Figura 2. Especímenes de Drymarchon corais preservados. FHG0 5742 A: cuerpo vista dorsal, B: cabeza vista lateral. FHGO 9091 C: cuerpo vista lateral, D: cabeza vista lateral.

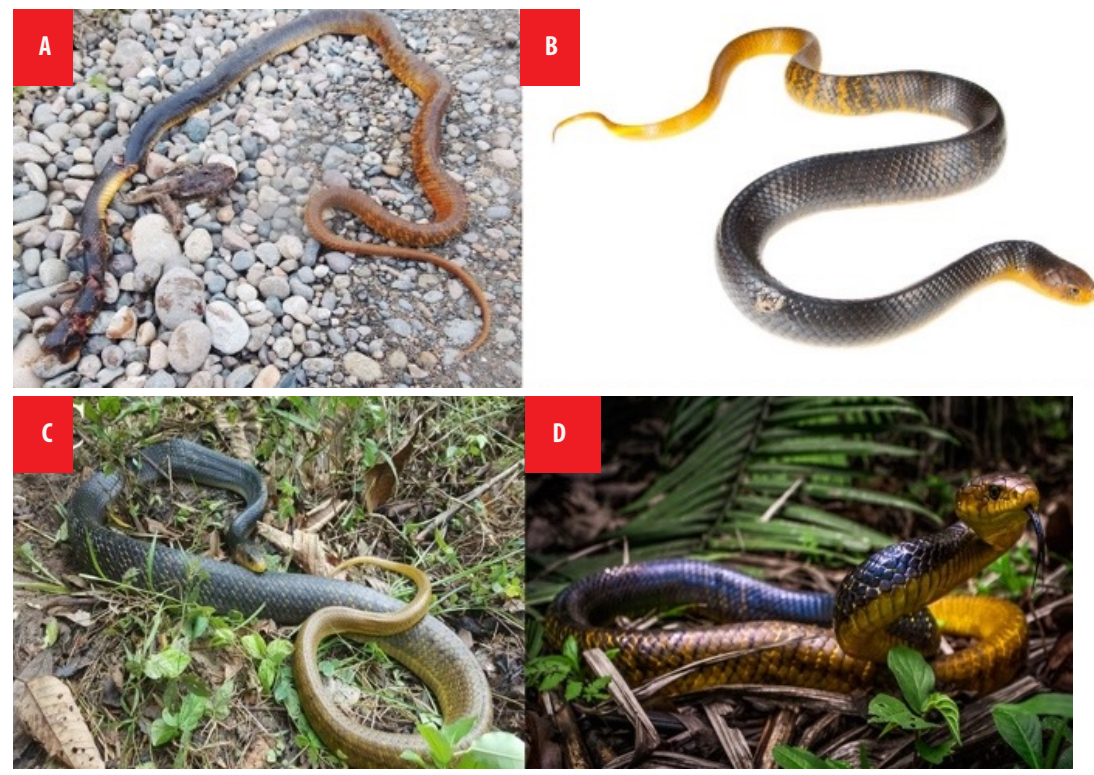




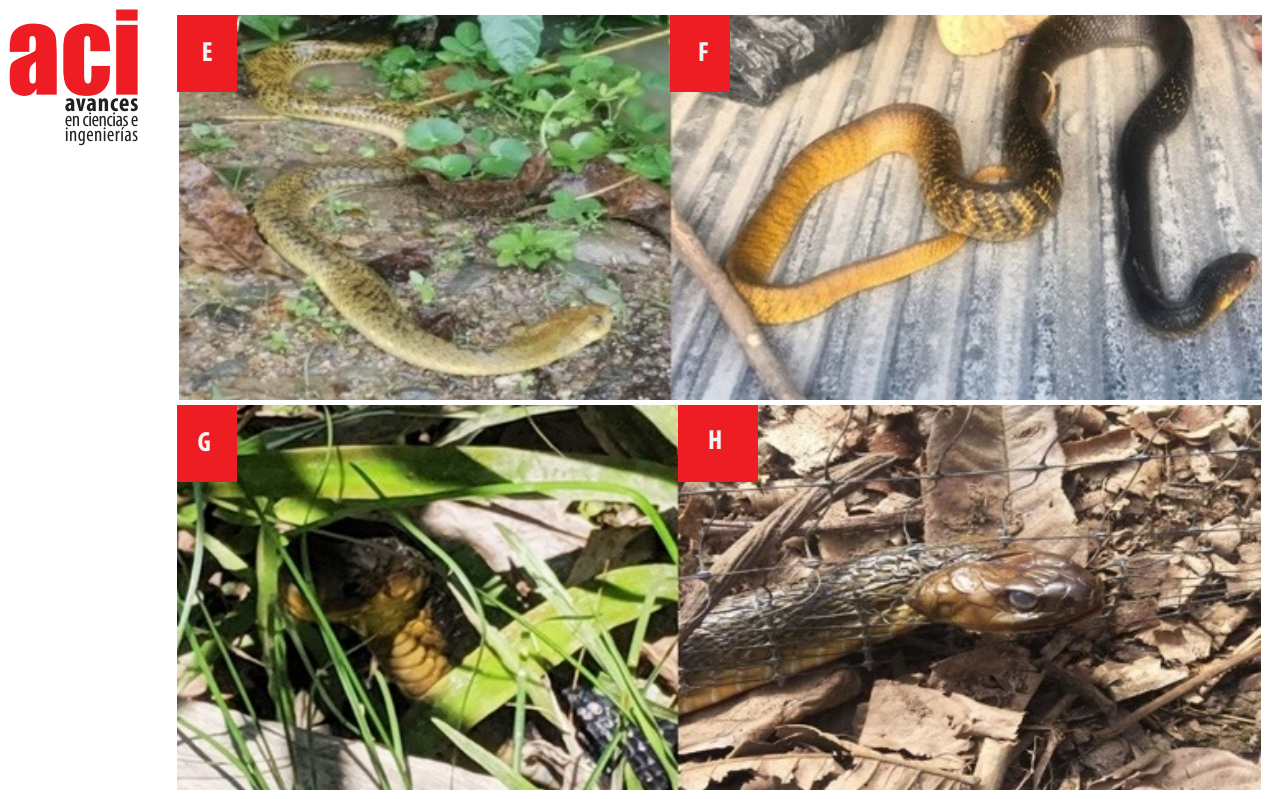

Figura 3. Registros fotográficos de la historia natural y nuevas localidades de Drymarchon corais en la Amazonía ecuatoriana. A: predación de Rhinella marina Sucumbíos, comunidad 13 de Marzo B: Napo, Ahuano. C: Orellana, Chiru Isla. D: Morona Santiago, Cangaime. E: Morona Santiago, Guayabal. F: Orellana, Nueva Esperanza. G: Orellana, Nuevo Rocafuerte. H: Zamora Chinchipe, Nuevo Paraíso.

Los dos especímenes colectados (FHGO 5742 y FHGO 9091) y depositados en el museo de Anfibios y Reptiles de la Fundación Herpetológica Gustavo Orcés fueron identificados como Drymarchon corais por concordar con los siguientes caracteres propuestos por Wüster et al. [13], Boie [35]; Pérez-Santos y Moreno [23]: "(1) menor tamaño que D. melanurus; (2) mancha de gran tamaño con tres ramificaciones negras debajo del ojo presente; (3) 19-17-15 dorsales; (4)188-216 ventrales; (5) 66-81 subcaudales; (6) cambio de color a lo largo del dorso de los adultos, más oscuro anteriormente y pálido posteriormente; (7) marca oblicua negra al lado del cuello ausente; (8) color uniforme y pálido a lo largo de la región ventral y la cola; (9) subcaudales oscuras o pálidas ausentes".

Observaciones adicionales reportan una hembra adulta enrollada sobre un talud al borde de la carretera, entre los helechos, a la entrada de la Estación Científica de la Fundación Jatun Sacha ( $1^{\circ} 4^{\prime} 0,84^{\prime \prime}$ S; 77³7'12,72" O; 427 m s.n.m.), parroquia Ahuano, cantón Tena, provincia de Napo, el 07 de noviembre de 1999. Dos individuos adultos fueron observados en la Estación de Biodiversidad Tiputini, cantón Aguarico, provincia de Orellana. El primero el 29 de diciembre de 1999, moviéndose durante el día entre las ramas de un árbol a $5 \mathrm{~m}$ del borde del río Tiputini $\left(0^{\circ} 38^{\prime} 15,72^{\prime \prime} \mathrm{S}\right.$; 76²'59,64" O; $216 \mathrm{~m}$ s.n.m.) y el segundo el 12 de junio de 2000 mientras se movía entre las raíces tabulares de un ceibo $\left(0^{\circ} 38^{\prime} 22,20^{\prime \prime} \mathrm{S} ; 76^{\circ} 9^{\prime} 38,16^{\prime \prime} \mathrm{O} ; 223 \mathrm{~m}\right.$ s.n.m.). Un cuarto individuo adulto fue observado en el suelo cerca a la comunidad Nueva Juventud $\left(0^{\circ} 4^{\prime} 43,00^{\prime \prime} \mathrm{S} ; 76^{\circ} 24^{\prime} 9,00^{\prime \prime}\right.$ O; 249 m s.n.m.), parroquia Tarapoa, cantón Cuyabeno, provincia de Sucumbíos el 15 de julio de 2000 (D. Cisneros-Heredia in litt. 2020). 
Cuatro ejemplares de Drymarchon corais (J. Culebras in litt. 2020) fueron reportados con los siguientes datos: (1) un individuo adulto activo a las 12:00 h, el 04 de febrero de 2012, en un día soleado en un bosque conservado en el sector de Limoncocha (0²2'48,30" S; 76³5'36,73" O; 251 m s.n.m.), provincia de Sucumbíos; (2) un individuo activo a las 11:30 h, el 10 de noviembre de 2016 en la Estación de Biodiversidad Tiputini (0³8'10,32" S; 769'51,99" O: 218 m s.n.m.), provincia de Orellana; (3) un individuo capturado y fotografiado el 14 de junio de 2017 en un área alterada en el sector de Ahuano (1³'13,46" S; 77³1'51,34" O; 356 m s.n.m.), provincia de Napo (Fig. 3B); (4) un individuo activo a las 10:00 h en un día soleado en Kapawi (2³2'28,24" S; 7651'30,96" O; 249 m s.n.m.), provincia de Pastaza.

Seis individuos de D. corais fueron reportados en el cantón Zamora, provincia de Zamora Chinchipe por D. Núñez con los siguientes datos: (1) el 25 de abril de 2012 a las 13:35 h un individuo cruzando una vía cercana al río Bombuscaro en dirección al Parque Nacional Podocarpus (404'04,61" S; 7856'23,27" O; 937 m s.n.m.); (2) el 07 de agosto de 2014 un individuo durmiendo en un árbol a $1.80 \mathrm{~m}$ del suelo en una franja de bosque intervenido en la vía a Jenairo ( $4^{\circ} 2^{\prime} 57,29^{\prime \prime} \mathrm{S} ; 78^{\circ} 58^{\prime} 0,20^{\prime \prime}$ O; 1048 m s.n.m.); (3) el 15 de enero de 2015, observó un individuo a las 10:15 h cruzando una vía de segundo orden en un parche de bosque intervenido junto al estadio municipal Reina del Cisne (4 $4^{\circ} 13,01^{\prime \prime} \mathrm{S} ; 78^{\circ} 56^{\prime} 27,21^{\prime \prime}$ O; 960 m s.n.m.); (4) el 22 de marzo de 2015 a 15:15 h, un individuo muerto en una vía

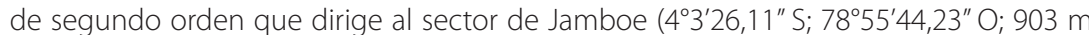
s.n.m.); (5) el 13 de julio de 2018 a las 09:55 h un individuo muerto en una vía colindante con una zona de potrero en el cantón Nangaritza, parroquia Congüime $\left(4^{\circ} 2^{\prime} 53,34^{\prime \prime} \mathrm{S}\right.$; $78^{\circ} 38^{\prime} 9,12^{\prime \prime}$ O; 896 m s.n.m.) y (6) el 17 de noviembre de 2018, un individuo durmiendo en un árbol a 2 m del suelo junto al río Chuchumbletza ( $3^{\circ} 34^{\prime} 18,85^{\prime \prime}$; $78^{\circ} 31^{\prime} 16,97^{\prime \prime} \mathrm{O} ; 751$ m s.n.m.), vía a Tundayme, cantón El Pangui en el límite entre las provincias de Zamora Chinchipe y Morona Santiago.

Un individuo adulto, con longitud total de $270 \mathrm{~cm}$, fue capturado y fotografiado en la zona de amortiguamiento del Parque Nacional Yasuní cerca al río Huarmi Yuturi en el

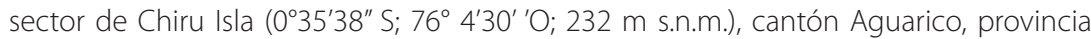
de Orellana (Fig. 3C), el 06 de mayo de 2018 a las 10:48 h. El individuo fue observado desplazándose sobre un pantano en un área intervenida compuesta por bosque en regeneración, áreas abiertas y abundante vegetación herbácea (L. Oyagata in litt. 2019).

Otro individuo adulto con una longitud aproximada de $200 \mathrm{~cm}$ fue observado y fotografiado en una comunidad Shuar cercana al río Cangaime $\left(2^{\circ} 47^{\prime} 15^{\prime \prime} \mathrm{S} ; 77^{\circ} 31^{\prime} 33^{\prime \prime} \mathrm{O}\right.$; 224 m s.n.m.), sector Puerto Morona, cantón Tiwintza, provincia de Morona Santiago (Fig. 3D), el 27 de mayo de 2019 por Axel Marchelie. El individuo fue encontrado a las 08:00 h tomando el sol sobre el suelo de un pastizal a $20 \mathrm{~m}$ de un pequeño arroyo. Inicialmente, el individuo intentó huir lentamente hacia un agujero debajo de las raíces de un árbol de chonta (Bactris gasipaes). En el intento de captura la serpiente se mostró muy agresiva intimidando con la boca abierta e intentando morder por dos ocasiones a la altura de las rodillas.

Un individuo adicional, de aproximadamente $250 \mathrm{~cm}$ fue observado y fotografiado en el

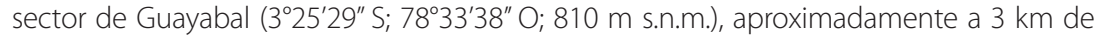
Gualaquiza, provincia de Morona Santiago (Fig. 3E), el 09 de abril de 2020 a las 07:23 h por 
Ángel Vásquez. El individuo fue observado mientras se desplazaba entre la vegetación herbácea de una pequeña poza en medio de una zona de pastizal en una finca.

La información contemplada en la plataforma digital iNaturalist señala que; (1) Drymarchon corais es una especie reportada por Diego Naranjo el 19 de junio de 2017 en la parroquia de Nueva Esperanza (0²9'57,48' S; 76²5'53,80" O; 261 m s.n.m.), provincia de Orellana (Fig. 3F); (2) el 07 de agosto de 2019 reportada por Mike Tidwell en la parroquia de Nuevo Rocafuerte, cantón Aguarico, provincia de Orellana (055'22,36" S; 75²3'57,64" O; 183 m s.n.m.) (Fig. 3G); (3) el 16 de febrero de 2020 en la parroquia Ahuano, cantón Tena, provincia de Napo ( $1^{\circ} 04^{\prime} 36,50^{\prime \prime}$ S; 77³1'40,53" O; 433 m s.n.m.); y (4) el 20 de abril de 2020 reportado un individuo muerto en la parroquia Nuevo Paraíso, cantón Nangaritza, provincia de Zamora Chinchipe (4²8'03,93" S; 78²41'26,95" O; 1110 m s.n.m.) (Fig. 3H).

\section{DISCUSIÓN}

Los registros de las nuevas localidades presentadas para la serpiente índigo Drymarchon corais en las provincias de Sucumbíos, Pastaza, Morona Santiago y Zamora Chinchipe son los primeros oficialmente documentados y reportados en la Amazonía ecuatoriana. Nuestros registros incluyen latitudinalmente los puntos más cercanos y conocidos de la especie hacia las fronteras con Colombia (al Norte) y Perú (al Sur). Estos registros amplían el rango de distribución geográfica conocida de la especie en aproximadamente 65 $\mathrm{km}$ hacia el Norte y $511 \mathrm{~km}$ hacia el Sur desde sus localidades previamente conocidas $[4,18,22]$ dando un área total de ocupación de la especie de 28.940,81 km². Además, reportamos individuos observados, fotografiados e identificados sobre los $1110 \mathrm{~m}$ s.n.m. en la provincia de Zamora Chinchipe, siendo estos los registros más altos para esta especie ampliando el rango altitudinal conocido previamente en la Amazonía ecuatoriana de $0-230 \mathrm{~m}$ a $0-1100 \mathrm{~m}$ s.n.m. $[4,18,22]$.

Los datos de historia natural de Drymarchon corais indican alta tolerancia hacia ambientes antrópicos en concordancia con varios autores [14,16,27,29,36,37]. Basados en nuestros registros reconfirmamos en parte lo expuesto por Wüster et al. [13]; Bernade y Abe [14]; Da Costa et al. [16]; McCranie, [27] quienes mencionan que la especie es diurna, con un forrajeo activo en el estrato terrestre y con alta afinidad hacia los cuerpos de agua [27]. Por otra parte, el registro de varios individuos de D. corais en el estrato arbóreo incrementaría la lista de especies que erróneamente han sido identificadas únicamente con hábitos terrestres [38]. Adicionalmente, identificamos que D. corais presenta un comportamiento agresivo y territorial cuando tiene encuentros ocasionales con las personas. Es probable que esta agresividad y su gran tamaño signifiquen un factor adverso para su conservación ya que estas características tienden a producir mayor temor en la gente $[39,40]$.

Estudios históricos desarrollados en otros países determinaron que D. corais era abundante en áreas protegidas con buen hábitat [41]. Sin embargo, a partir de la década de los 90 se evidenció el declive de sus poblaciones [42]. Una situación similar ocurre en la Amazonía ecuatoriana, donde por años, se han realizado diversos estudios que han incluido colecciones científicas, pero al parecer la especie es bastante esquiva a 
los muestreos además de poco abundante [24,43]. Lo señalado concordaría con las categorías de la IUCN [44], donde globalmente Drymarchon corais está categorizada con Preocupación menor (LC) y localmente, en Ecuador, en Datos insuficientes (DD) [45]. Los nuevos registros de la especie presentados para todas las provincias de la Amazonía ecuatoriana, sugerirían que su estado poblacional es estable. Sin embargo, es necesario focalizar estudios y esfuerzos conjuntos de conservación para identificar las principales amenazas de la especie y determinar el estado real de sus poblaciones. Finalmente, y no menos importante, se considera que una campaña agresiva de educación ambiental sería una estrategia de conservación vital que permitiría generar una mayor oportunidad de supervivencia de la especie.

\section{AGRADECIMIENTOS}

A Tashkin Meza por la logística y ayuda en el trabajo de campo. A Katty Garzón-Tello (FHGO) por permitirnos el acceso a sus colecciones. A Mónica Guerra (MEPN) por compartirnos la información de la colección a su cargo. A Mario H. Yánez-Muñoz y los dos revisores anónimos por los acertados comentarios que permitieron mejorar el manuscrito. A Diego Cisneros-Heredia, Jaime Culebras, Axel Marchelie, Luis Oyagata, Darwin Núñez y Ángel Vásquez por facilitarnos la información de sus registros y fotografías de los especímenes.

\section{CONTRIBUCIONES DE LOS AUTORES}

Manuel R. Dueñas: diseño de la metodología en campo, toma de datos, colección de espécimen, revisión de material colectado, diseño de la investigación, revisión de literatura, redacción del manuscrito, respuesta de observaciones. Jorge $\mathrm{H}$. Valencia: diseño de la investigación, revisión de literatura, redacción del manuscrito. Daniela Franco-Mena: búsqueda de información, revisión de literatura y redacción del manuscrito.

\section{CONFLICTO DE INTERÉS}

Los autores declaramos no tener ningún conflicto de intereses en el desarrollo de la presente investigación. 


\section{REFERENCIAS}

[1] Brown, J. H. (2014). Why are there so many species in the tropics? Journal of Biogeography, 41:8-22. doi: https:// doi.org/10.1111/jbi.12228

[2] Andresen, E., Arroyo-Rodríguez, V., \& Escobar, F. (2018). Tropical biodiversity: the importance of biotic interactions for its origin, main-tenance, function, and conservation. In: Dáttilo, W., Rico-Gray, V. (Eds) Ecological networks in the tropics. Springer, Cham, 1-13. doi: https://doi.org/10.1007/978-3-319-68228-0_1

[3] Varela, L. A., \& Ron, S. R. (2018). Geografía y clima del Ecuador. BIOWEB. Pontificia Universidad Católica del Ecuador. Disponible en https://bioweb.bio/geoclima/ Consulta: 18 de junio de 2021.

[4] Torres-Carvajal, 0., Pazmiño-Otamendi, G., \& Salazar-Valenzuela, D. (2020). 19 de junio de 2021. Reptiles del Ecuador. Versión 2020.0. Museo de Zoología, Pontificia Universidad Católica del Ecuador. https://bioweb.bio/ faunaweb/reptiliaweb

[5] Yánez-Muñoz, M. H., Bejarano-Muñoz, P., Brito, J., \& Batallas, D. (2014). Ranas terrestres de los Andes Surorientales de Ecuador II: Una nueva especie de Pristimantis verde espinosa de los bosques montanos del Parque Nacional Sangay (Anura: Craugastoridae). Avances en Ciencias e Ingenierías, 6(2), 63-77. doi: https://doi.org/10.18272/aci. v6i2.180

[6] Valencia, J. H., Dueñas, M. R., Székely, P., Batallas, D., Pulliquitín, F.\& Ron, S. (2017). A new species of directdeveloping frog of the genus Pristimantis (Anura: Terrarana: Craugastoridae) from Cordillera del Cóndor, Ecuador with comments on threats to the anuran fauna of the region. Zootaxa, 4353(3), 447-466. doi: https://doi.org/10.11646/ zootaxa.4353.3.3

[7] Valencia, J. H., Valladares-Suntasig, F., Tipantiza-Tuguminago, L., \& Dueñas, M. R. (2019). A new species of terrestrialbreeding frog of the genus Pristimantis (Anura: Terrarana: Craugastoridae) from the eastern Andean slopes of the southern Ecuador. Zootaxa, 4658(3), 509-525. doi: https://doi.org/10.11646/zootaxa.4658.3.4

[8] Dueñas, M. R., Valencia, J. H., Yánez-Muñoz, M. H., \& Eguiguren, J. S. (2018). Primer registro de la salamanquesa asiática Hemidactylus frenatus Duméril \& Bribon, 1834 (Sauria: Gekkonidae) en la Cordillera del Cóndor, Zamora Chinchipe, Ecuador. Cuadernos de herpetología, 32(1).

[9] Ortega-Andrade, H. M., \& Valencia, J. H. (2010). First country records of Pristimantis luscombei (Duellman and Mendelson) and Syncope tridactyla (Duellman and Mendelson) in eastern lowlands of Ecuador (Amphibia: Anura: Strabomantidae, Microhylidae). Herpetology Notes, 3, 251-256.

[10] Yánez-Muñoz, M. H., Toral-Contreras, E., Meza-Ramos, P., Reyes-Puig, J. P., Bejarano-Muñoz, E. P., Mueses-Cisneros, J. J., \& Paucar, D. (2012). New country records for five species of Pristimantis Jiménez de la Espada, 1870 from Ecuador. Check List, 8(2), 286-290. doi: https://doi.org/10.15560/8.2.286

[11] Tipantiza-Tuguminago, L., Auqui, E., Dueñas, M. R., \& Valencia, J. H. (2018). First record of Pristimantis rufioculis (Duellman y Pramuk, 1999), from Ecuador. Herpetozoa, 31, 1-2.

[12] França, F. G. R., \& Araújo, A. F. (2006). The conservation status of snakes in central Brazil. South American Journal of Herpetology, 1(1), 25-30. doi: https://doi.org/10.2994/1808-9798(2006)1[25:TCSOSI]2.0.C0;2

[13] Wuster, W., Yrausquin, J. L., \& Mijares-Urrutia, A. (2011). A new species of indigo snake from north-western Venezuela (Serpentes: Colubridae: Drymarchon). Herpetological Journal, 11(4), 157-166.

[14] Bernarde, P. S., \& Abe, A. S. (2006). A snake community at Espigão do Oeste, Rondônia, southwestern Amazon, Brazil. South American Journal of Herpetology, 1(2), 102-113. doi: https://doi.org/10.2994/18089798(2006)1[102:ASCAED]2.0.C0;2

[15] Campos, V. A., Oda, F. H., Curcino, A., F., \& Curcino, A. (2010). An unusual prey item for the yellow tail cribo Drymarchon corais Boie 1827, in the Brazilian Savannah. Herpetology Notes, 3, 229-231.

[16] Costa-Prudente, A. L., Costa Mensk, A., Magalhães da Silva, F., \& Maschio, G. F. (2014). "Diet and reproduction of the Western Indigo Snake Drymarchon corais (Serpentes: Colubridae) from the Brazilian Amazon". Herpetological Notes, 7,99-108. 
17] Pelegrini, J. S., Venâncio, N. M., \& Kuniy, A. (2019). Note on an event of double predation: Leptodeira annulata (LINNAEUS, 1758) (Serpentes: Dipsadidae) being predated by Drymarchon corais BOIE, 1827 (Serpentes: Colubridae), being predated in turn by Bothrops atrox (LINNAEUS, 1758) (Serpentes: Viperidae). Herpetology Notes, 12, 1193-1195. doi: https://www.jstor.org/stable/24530016

[18] Rodríguez-Guerra, A., \& Mármol-Guijarro, A. (2019). Drymarchon corais En: Torres-Carvajal, 0., Pazmiño-Otamendi, G. \& Salazar-Valenzuela, D. (2019). Reptiles del Ecuador. Versión 2019.0., acceso Jueves, 30 de Julio de 2020 Museo de Zoología, Pontificia Universidad Católica del Ecuador. https://bioweb.bio/faunaweb/reptiliaweb/FichaEspecie/ Drymarchon\%20corais.

[19] Leynaud, G. C., \& Bucher, H. (1999). La fauna de serpientes del Chaco sudamericano: diversidad, distribución geográfica y estado de conservación. Vol. 98. Córdoba: Academia Nacional de Ciencias.

[20] Wallach, V. K., Williams, L., \& Boundy, J. (2014). Snakes of the world: a catalogue of living and extinct species. CRC press.

[21] Uetz, P., Freed, P, Aguilar, R. \& Hošek, J. (eds.) (2021) The Reptile Database, http://www.reptile-database.org, accessed on 08 June 2021.

[22] Cisneros-Heredia, D. F. (2006). La herpetofauna de la Estación de Biodiversidad Tiputini, Ecuador. Diss. BS Thesis. Quito, Ecuador: Universidad San Francisco de Quito.

[23] Pérez-Santos, C., Moreno, A. G. (1991). Serpientes de Ecuador. Vol. 11. Museo Reg. Scienze Nat. Torino.

[24] Ortega-Andrade, H. M. (2010). Diversidad de la Herpetofauna en la Centro Amazonía del Ecuador (Doctoral dissertation, Tesis para obtener el grado de Maestro en Ciencias. Biología y Conservación de Vertebrados. Xalapa, Veracruz, México. Instituto de Ecología AC). 1-150.

[25] Amaral, A. (1929). "Estudos sobre ofídios neotrópicos. XXI. Revisão do gênero Drymarchon Fitzinger" Memorias do Instituto Butantan, 4,323-330

[26] Smith, H., M. (1941). A review of the subspecies of the indigo snake (Drymarchon corais). Journal of the Washington Academy of Sciences, 31(11), 466-481.

[27] McCranie, J., R. (1980). Drymarchon Fitzinger Indigo snake. Catalogue of American Amphibians and Reptiles, 267, $1-4$.

[28] Parker, H. W. (1938). Ll. - The vertical distribution of some reptiles and Amphibians in Southern Ecuador" Annals and Magazine of Natural History, 2(11), 438-450. doi: https://doi.org/10.1080/00222933808526872

[29] Miyata, K. (1982). A check list of the amphibians and reptiles of Ecuador with a bibliography of Ecuadorian herpetology. Smithsonian Herpetological Information Service. doi: https://doi.org/10.5479/si.23317515.54.1

[30] Almendáriz, A. "Lista de vertebrados del Ecuador. Anfibios y reptiles" 1991

[31] Valencia, J. H., Toral, E., Morales, M., Betancourt-Yépez, R. \& Barahona, A. (2008). Guía de campo reptiles del Ecuador. Fundación Herpetológica Gustavo Orcés, Simbioe Quito, Ecuador. 236 pp.

[32] Ines Hladki, A., Ramírez Pinilla, M., Renjifo, J., Urbina, N., Nogueira, C., Gonzales, L., Catenazzi, A., Cisneros-Heredia, D.F., Hoogmoed, M., Schargel, W., Rivas, G. \& Murphy, J. 2019. Drymarchon corais. The IUCN Red List of Threatened Species 2019: e.T62234A3110201. doi: https://dx.doi.org/10.2305/UUCN.UK.2019-3.RLTS.T62234A3110201.en. Downloaded on 05 January 2020

[33] Valencia, J. H., Garzón-Tello, K., Tipantiza-Tuguminago, L., Pulliquitín, F., Barragán-Paladines, \& Noboa, G. (2017). Serpientes del Distrito Metropolitano Quito (DMQ), Ecuador, con comentarios sobre su rango geográfico y altitudinal y conservación. Avances en ciencias e ingenierias, 9(15), 19-60. doi: http://dx.doi.org/10.18272/aci.v9i15.305

[34] GBIF.org (13 July 2020) GBIF Occurrence Download. doi: https://doi.org/10.15468/dl.v85t3c

[35] Boie, F. von. (1827) "Bemerkungen über Merrem's Versuch eines Systems der Amphibien, 1. Lieferung: Ophidier." Isis van 0 ken, $20,508-566$.

[36] Hoogmoed, M. S., Dixon, J. R., \& Soini, P. (1986) The reptiles of the upper Amazon basin, Iquitos region, Peru, I-VII. Milwaukee Public Museum. Amphibia-Reptilia, 9(3), 315-317.

[37] Simmons, J. E. (2002). Herpetological collecting and collections management. Salt Lake eUtah Utah: Society for the Study of Amphibians and Reptiles. 
[38] Dueñas, M. R., \& Valencia, J. H. (2018). Bothrocophias microphthalmus (Small-eyed Toad-headed Pitviper). Habitat Use and Diet. Herpetological Review, 49(3), 542.

[39] Greene, H. W., \& Campbell, J. A. (1992). The future of pitvipers. Biology of the Pitvipers, 421-427.

[40] Lynch, J. D. (2012). El contexto de las serpientes de Colombia con un análisis de las amenazas en contra de su conservación. Revista de la Academia Colombiana de Ciencias Exactas, Físicas y Naturales, 36(140), 435-449.

[41] U. S Fish and Wildlife Service. (1982). Eastern indigo snake recovery plan. U.S Fish and Widdlife Service, Atlanta, GA.

[42] Hallam, C. 0., Wheaton, K., \& Fischer, R. A. (1998). Species Profile: Eastern Indigo Snake (Drymarchon corais couperi) on Military Installations in the Southeastern United States. Strategic Environmental Research and Development Program.

[43] Duellman, W. E. (1978). The biology of an equatorial herpetofauna in Amazonian Ecuador. Miscellaneous Publications of the University of Kansas, $65,1-352$.

[44] IUCN, 2020. The IUCN Red List of Threatened Species. Version 2020-1. [cited 14 March 2020] https://www.iucnredlist. org. (March 2020).

[45] Carrillo, E., Aldás, S., Altamirano-Benavides, M. A., Ayala-Varela, F., Cisneros-Heredia, D. F., Endara, A., Márquez, C., Morales, M., Nogales-Sornosa, F., Salvador, P., Torresm M. L., Valencia, J., Villamarín-Jurado, F., Yánez-Muñoz, M. H. \& Zárate, P. (2005). Lista roja de los reptiles del Ecuador. Fundación Novum Milenium, UICN-Sur, UICN ComitéEEcuatoriano, Ministerio de Educación y Cultura, Serie Proyecto Peepe, Quito, Ecuador, 1-46. 